\title{
MÉTRICA PARA LA GESTIÓN DE LA SOSTENIBILIDAD ECONÓMICA, EN SISTEMAS PRODUCTIVOS DE CACAO
}

\section{METRIC FOR THE MANAGEMENT OF ECONOMIC SUSTAINABILITY, IN COCOA PRODUCTION SYSTEMS}

MÉTRICA PARA A GESTÃO DA SUSTENTABILIDADE ECONÔMICA, NOS SISTEMAS DE PRODUÇÃO DE CACAU

\author{
Alberto Ducuara Manrique', \\ Armando Torrente Trujillo². \\ Grupo de investigación PYMES, \\ Universidad Surcolombiana.
}

\begin{abstract}
1 Doctorando en Agroindustria y Desarrollo Agrícola Sostenible en la Universidad Surcolombiana. Ingeniero Industrial de la Universidad INCCA de Colombia, Especialista en Alta Gerencia de la Universidad Surcolombiana y en Gerencia de Mercadeo de la Universidad de la Salle. Magíster en Historia de la Universidad Nacional de Colombia. Docente Investigador de planta de la Universidad Surcolombiana, grupo Pymes de la Facultad de Economía y Administración, de la Universidad Surcolombiana de Neiva -Huila - Colombia, https://orcid.org/0000-0001-8869-7428 alduma@usco.edu.co

$2 \quad$ Doctorado en Ciencias Agropecuarias en la Universidad Nacional de Colombia, Ingeniero Agrícola de la Universidad Nacional de Colombia, Magíster en Ciencias Agrarias de la Universidad Nacional de Colombia, Especialista en Riego de la Universidad Surcolombiana. Docente Investigador de planta de la Universidad Surcolombiana, grupo GHIDA Facultad de Ingeniería, de la Universidad Surcolombiana de Neiva - Huila - Colombia https://orcid. org/0000-0001-6656-4624 armando.torrente@usco.edu.co
\end{abstract}

\section{RESUMEN}

Este artículo hace parte del trabajo investigativo, para determinar un modelo metodológico, que facilite monitorear y evaluar la gestión de la sostenibilidad económica en plantaciones de cacao en el Huila. Allí se plantea como objetivo principal, diseñar un modelo cuantitativo, que oriente la gestión eficiente en sistemas productivos de cacao, a partir de un conjunto de indicadores, por parte de los productores, bajo una racionalidad y motivación específica. 
Las investigaciones científicas analizan variables y en un sistema productivo de cacao, encontramos diversas variables de insumo, proceso y resultado. A partir de este principio y desde una perspectiva ecléctica, se pretende encontrar una metodología, centrada en métricas con indicadores de sostenibilidad.

En este sentido, se propone el empleo de una estructura jerárquica para la selección de indicadores, adaptada de la Teoría de Principios, Criterios e Indicadores PC\&I (Lammerts van Bueren \& Blom, 1996). El proceso de investigación en trámite, prevé realizar una validación empírica, en sistemas productivos de cacao de la zona norte del departamento del Huila. El modelo metodológico a desarrollar, debe servir como herramienta, para el proceso de toma de decisiones del productor y de las instituciones, a nivel local, regional o global.

PALABRAS CLAVE, modelo, sostenibilidad, indicadores, cacao, gestión

\section{SUMMARY}

This article is part of the research work to determine a methodological model that facilitates monitoring and evaluating the management of economic sustainability in cocoa plantations in Huila. There it is proposed as a main objective, to design a quantitative model, which guides the efficient management in cocoa production systems, from a set of indicators, by the producers, under a rationality and specific motivation.

Scientific research analyzes variables and in a cocoa production system, we find various variables of input, process and result. From this principle and from an eclectic perspective, it is intended to find a methodology, focused on metrics with sustainability indicators.

In this sense, it is proposed to use a hierarchical structure for the selection of indicators, adapted from the Theory of Principles, Criteria and
Indicators (Lammerts van Bueren \& Blom, 1996) PC\&I. The research process in process, plans to carry out an empirical validation in cocoa production systems in the northern area of the department of Huila. The methodological model to be developed should serve as a tool for the decision-making process of the producer and institutions, at the local, regional or global level.

KEYWORDS model, sustainability, indicators, cocoa, management

\section{RESUMO}

Este artigo faz parte do trabalho de pesquisa para determinar um modelo metodológico que facilite o monitoramento e a avaliação da gestão da sustentabilidade econômica nas plantações de cacau em Huila. Lá é proposto como objetivo principal, projetar um modelo quantitativo, que orienta a gestão eficiente nos sistemas de produção de cacau, a partir de um conjunto de indicadores, pelos produtores, sob racionalidade e motivação específica.

Pesquisas científicas analisam variáveis e em um sistema de produção de cacau, encontramos várias variáveis de insumo, processo e resultado. A partir desse princípio e de uma perspectiva eclética, pretende-se encontrar uma metodologia, focada em métricas com indicadores de sustentabilidade.

Nesse sentido, propõe-se utilizar uma estrutura hierárquica para a seleção de indicadores, adaptada da Teoria dos Princípios, Critérios e Indicadores (Lammerts van Bueren \& Blom, 1996)PC\&I. O processo de pesquisa, planeja realizar uma validação empírica em sistemas de produção de cacau na zona norte do departamento de Huila. O modelo metodológico a ser desenvolvido deve servir como ferramenta para o processo de tomada de decisão do produtor e das instituições, a nível local, regional o global. 


\section{PALAVRAS-CHAVE}

modelo, sustentabilidade, indicadores, cacau, gestão

\section{INTRODUCCIÓN}

Los conceptos teóricos consultados, para poder comprender de manera integral las problemáticas relacionadas con la sostenibilidad agraria en general y particular la del cacao, prevén un enfoque multiescalar, mediante relaciones jerárquicas, propias de los sistemas de producción, funcionando en un marco de distintos niveles espaciales y temporales; en donde se interrelacionan los procesos sociales y naturales, mediados por la racionalidad económica dominante, que giran alrededor de las instituciones y de las formas de organización que gobiernan las actividades agronómicas, susceptibles de ser medidos y evaluados con indicadores.

Con este propósito desde la Organización de Naciones Unidas 1999 y la OECD 2008, se han trazadolineamientos yguías, parala construcción de métricas que facilite la comprensión y evaluación de sistemas productivos agrarios, con indicadores. En sus informes, se reconoce que un indicador es una variable que brinda las bases para evaluar tendencias económicas, ambientales y sociales o establecer metas de políticas. Estos indicadores pueden ser cualitativos, cuantitativos, como también pueden ser índices compuestos por la relación entre diferentes variables (OECD \& JRC, 2008).

También se concluye que existen diversas concepciones respecto a la sostenibilidad. Esta situación demanda de los investigadores y las instituciones, metodologías y estándares, para crear habilidades de comprensión, a las interacciones entre naturaleza y sociedad, con trayectoria de sostenibilidad. Lo anterior implica: 1) una escala económica, 2) una distribución equitativa de recursos para el sostenimiento intergeneracional, 3) enfoque al capital natural. Todo delimitado por la relación entre demanda y producción de recursos (Torres, Rodriguez, \& Sanchez, 2004).

Se suele actuar desde el campo académico, definiendo los pilares que dividen (económico, social, ambiental, político, técnico) de alguna manera artificial, la sostenibilidad de un sistema productivo agrario. Bajo el enfoque de sistemas, se puede aterrizar esa realidad, para integrar lo económico con lo técnico y lo comercial y calcular un indicador de viabilidad económica del cacao.

Hay por lo tanto oportunidades para desarrollar herramientas de predicción del estado de los ecosistemas del cacao, que incluya los servicios ambientales y el capital natural, con enfoques específicos para problemáticas diferenciadas a nivel local, a partir de indicadores e índices. Como herramienta, permiten elaborar cálculos y probabilidades que cuantifican cambios, identifican procesos y proveen un marco para la evaluación y la toma de decisiones (Torres et al., 2004; Walz et al., 2000).

\section{MARCO REFERENCIAL}

El cacao, es un cultivo permanente, nativo de América (Cuba, 2017), originario del sotobosque, su genotipo proporciona las bases para la toma de decisiones acertadas en el mantenimiento y comportamiento agronómico. Para la ONU y la OCDE, el cacao es un cultivo amigable con el medio ambiente, connotación que facilita priorizar la gestión de la sostenibilidad. Bajo este enfoque, en la teoría del desarrollo sostenible se admite "tres pilares" en conjunto: la sociedad, la economía y el medio ambiente, considerados por la Comisión Bruntland (1987) como un proceso de cambio y como meta en la solución de los problemas de pobreza (Strange \& Bayley, 2012). 
Investigadores de la sostenibilidad agraria, como Douglass 1984; Conway y Barbier, 1990; Youlong y Smith, 1994; afirman en Sanchez (2009), que la existencia de una agricultura sostenible es condición sine qua non para lograr un desarrollo verdaderamente sostenible (Sanchez, 2009). Así mismo, existe consenso en definir que la sostenibilidad es la capacidad de satisfacer un conjunto de necesidades de forma continuada en el tiempo, con un carácter multidimensional por la provisión de alimentos, la protección medioambiental, la viabilidad económica y la aceptación social (Hansen, 1995).

Desde el enfoque económico, el concepto de sostenibilidad encuentra la influencia de la Globalización y el Desarrollo Local, teoría que la asocia a una fase del desarrollo del capitalismo y que no puede ser sino humano y sostenible; conceptos que corroboran Guimarães (1997), Buarque (1999), entre otros (Boisier, 2005).

La caracterización de la Sostenibilidad debe ser estocástica, con un tratamiento a la variabilidad como un determinante de la sostenibilidad y un componente de predicciones, Hansen et al., (1996). Expresar las predicciones en términos de probabilidades y temporalidades, es aplicable a los comportamientos de los pilares de la demanda como precios, oferta o rendimientos en el cacao. La caracterización de la Sostenibilidad debe ser diagnóstica y medible de manera integral e integrada.

En este sentido, el enfoque económico tiene un carácter dominante en la sostenibilidad del cacao. Por lo tanto, el proceso de toma de decisiones para el crecimiento y mejoramiento continuo a nivel del cultivo, se soporta en un análisis concreto sobre la cuota de mercado, barreras de entrada, rentabilidad financiera, calidad del grano seco, eficiencia en los costos, éxito en inversiones pasadas, financiación, evolución de los beneficios y fijación del precio.
Bajo la metodología SAFE (Sustainability Assessment of Farming and the Environment Framework) desarrollado por Sauvenier et al., (2006) y Van Cauwenbergh et al., (2007), la investigadora Gabriela Sánchez Fernández propone evaluar la sostenibilidad de la actividad agraria en tres escalas (sistema agrario, explotación agraria y parcela). Adaptó la teoría PC\&I (principios, criterios e indicadores) de Lammerts Van Bueren y Blom, 1997, a sistemas agrarios de Castilla y León en España y a partir de indicadores sintéticos, evaluó la sostenibilidad agraria en cultivos secanos y con riego (Sánchez et al., 2009).

El Marco de Evaluación del Manejo Sostenible de Tierras FESLM desarrollado por Smyth y Dumanski (1993) para la Organización de la Naciones Unidas para la Agricultura y la Alimentación (FAO), busca evaluar la sostenibilidad agraria, con énfasis en el deterioro de los suelos. Aquí se definen los indicadores que serán monitoreados, a partir de una estructura jerárquica en los niveles: productividad, seguridad, protección, viabilidad económica y aceptabilidad (Sánchez et al., 2009).

La medición de agroecosistemas a partir de indicadores, es un proceso que requiere de una construcción colectiva Acevedo (2003); Astier, et al., (2003); Delgado (2000); Moya, et al., (2002), Sandy, Castaño \& Grenier (1999); Castaño (1993), su metodología se basa en la experiencia, el conocimiento de las comunidades involucradas y grupos facilitadores, que contemplen un enfoque de investigación participativa mediante el cual se promueva el dialogo de saberes (Cárdenas, Giraldo, Idárraga, \& Vásquez, 2006).

Un modelo de alta aceptabilidad en América lo constituye el "Marco de Evaluación de la Sostenibilidad Basada en Indicadores" 
(MESMIS) (Masera, Astier, \& López, 2000), utilizada para explicar la sostenibilidad desde lo ambiental, social y económico. Este enfoque fue utilizado para evaluar la sostenibilidad del cacao en fincas del departamento de Norte de Santander Colombia y se considera un referente válido para la presente investigación (Ramirez, Sigarroa, \& Del Valle, 2014).

Estudios como los dirigidos por Meadows et al., 1992, sobre "Los límites del crecimiento" plantean la utilidad de medir los efectos negativos para el medio ambiente con indicadores, la extralimitación en la explotación agrícola a través de monocultivos, que califican como una consecuencia de la acción directa o indirecta de los seres humanos sobre los ecosistemas (Vilches, Gil, \& Toscano, 2014). Así mismo, con el enfoque de Desarrollo Sostenible, se propone la metodología de Investigación y Desarrollo del Sistema Agrícola FSR / D, que integra aspectos sociales, económicos, ambientales (Darnhofer, Gibbon, \& Dedieu, 2012).

Autores como Bonisoli, et al., (2018) citados por Barrezueta \& Paz (2018), identifican niveles de la sostenibilidad agrícola, a partir de la existencia de una relación directa entre las dimensiones económica y social con la naturaleza. Esta relación se logra con la construcción, deconstrucción y reconstrucción cotidiana, de experiencias pasadas y de cara a los posibles cambios en el futuro. Se sustenta en una propuesta empírica, soportada con un conjunto de indicadores de sostenibilidad económicos y sociales para comprobar los Índices de Sostenibilidad (IS) entre tipos de Cacao Nacional con sombrío y la variedad CCN51 en la provincia el Oro, Ecuador (Barrezueta \& Paz, 2018).

Un enfoque centrado en el "Marco de los Capitales de la Comunidad MCC", creó una metodología para evaluar sistemas agrícolas (Flora, Flora, \& Gasteyer, 2018), que reconoce a la comunidad rural inmersa en un conjunto de recursos susceptibles de reproducir, aunque se encuentre aislada o pobre. Cuando esos recursos (o activos) se invierten para crear nuevos recursos, se convierten en capital. Esta metodología divide los recursos en siete "capitales" (natural, cultural, humano, social, financiero, político y físico construido) para fomentar el análisis y la acción holística de la sostenibilidad a nivel sistemas productivos.

Como se observa, la oferta de modelos metodológicos para la sostenibilidad agrícola, es bastante amplia dada las condiciones espacio temporales, aunque no agota los requerimientos locales, con sus particularidades en oferta ambiental, cultura o mercado. En la mayoría de casos se le da gran importancia a la representación de la realidad a través de los modelos cualitativos y cuantitativos para cada situación. El objetivo de la presente investigación es precisamente la de proponer un modelo conceptual para una situación específica, la sostenibilidad económica del cacao.

\section{METODOLOGÍA}

La adopción de un enfoque de desarrollo operativo, para interpretar los diferentes indicadores, se prevé la aplicación de métodos de agregación de indicadores simples y herramientas versátiles como la técnica PC\&l, aplicada en diferentes áreas para asuntos específicos de sostenibilidad y el mejoramiento de los procesos operativos agrarios. El análisis multivariantea, es una estrategia de análisis integral, en sistemas complejos como el del cacao, que recibe presión del entorno cambiante con variables de demanda, oferta, precio y calidad, a nivel nacional e internacional, que se convierten en directrices para seleccionar indicadores de resultados a nivel de la plantación.

Las etapas para el cálculo de los índices de sostenibilidad y para la discusión de resultados, se enmarcan en el análisis multivariante, que permite crear nuevo conocimiento, a partir de 
organizar y agrupar todas las variables de un sistema en un conjunto de nuevas variables, obtenidas mediante la transformación de las iniciales, sin la pérdida significativa de la información (Ramirez, y otros, 2017). Es una secuencia de etapas, cuyo proceso permite identificar relaciones significativas de más de una variable de respuesta, con información obtenida mediante la observación y el análisis simultáneamente. Ver Gráfica No 1.

\section{Gráfica No 1 ETAPAS PARA EL ANÁLISIS DE LA SOSTENIBILIDAD ECONÓMICA DEL CACAO}

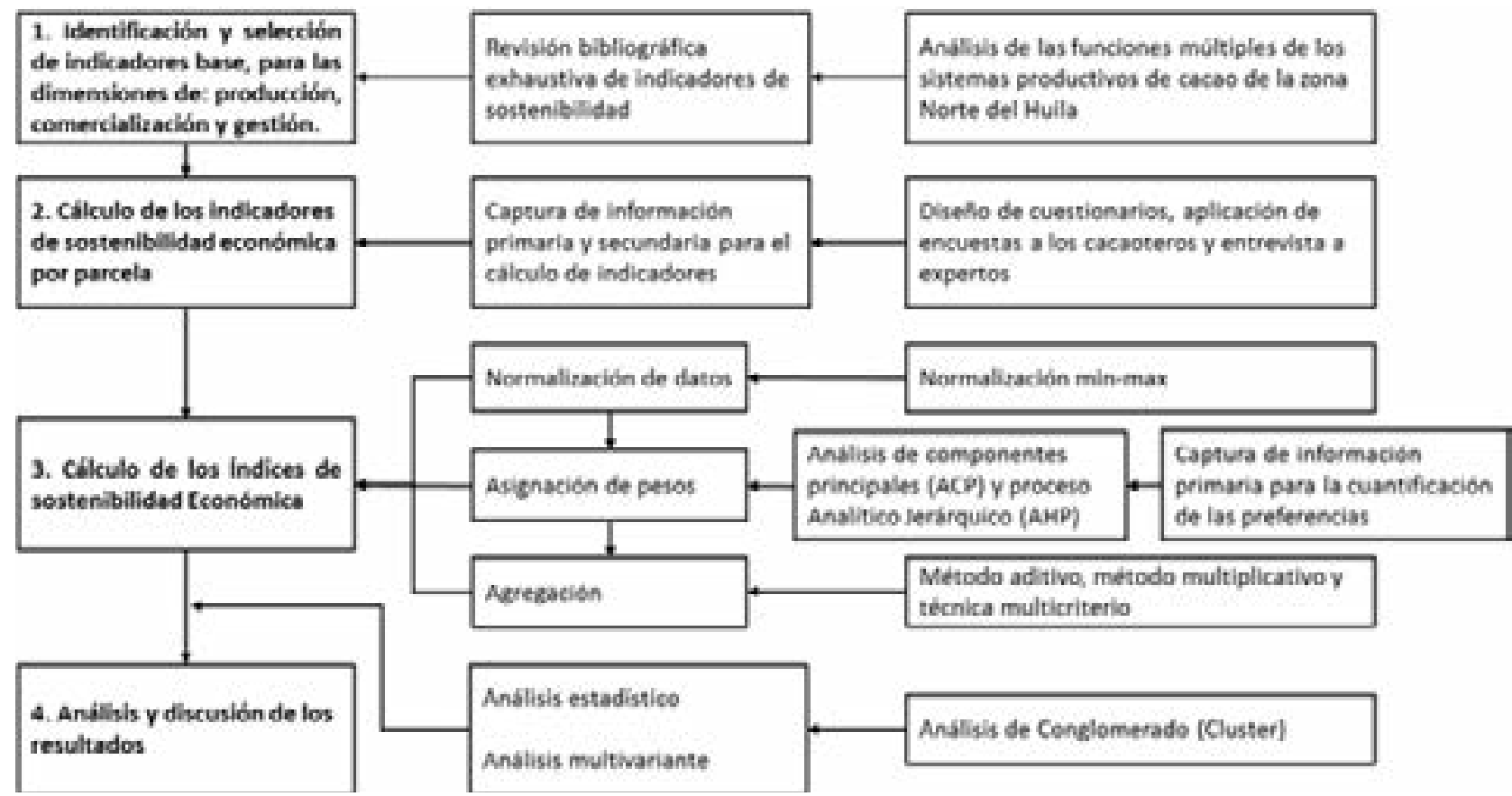

Fuente: Adaptado de Sánchez (2009)

\section{PC\&I PARA SELECCIONAR INDICADORES}

Con la determinación de variables, principios y criterio, se pueden definir constructos para ser medidos con indicadores de sostenibilidad. Los principios se formulan como una ley o norma fundamental en función de un objetivo, los criterios como parámetros de resultado y los indicadores como parámetros de resultado, proceso o insumo (Lammerts \& Blom et al., 1996). Los resultados a partir de indicadores de sostenibilidad, deben responder la pregunta de investigación y someter las hipótesis a prueba, utilizando como parte del diseño, las técnicas multivariantes (Hernandez, Fernández, \& Baptista, 2010).
Para desarrollar una respuesta concreta al concepto de sostenibilidad económica, su medición, monitoreo y evaluación, se describen a continuación, los principales elementos que precisamente ha guiado la selección de los indicadores, basado en una estructura jerárquica de principios y criterios. Ver Gráficas No 2 
Gráfica No 2 Principios, Criterios e Indicadores de Sostenibilidad Económica Seleccionados

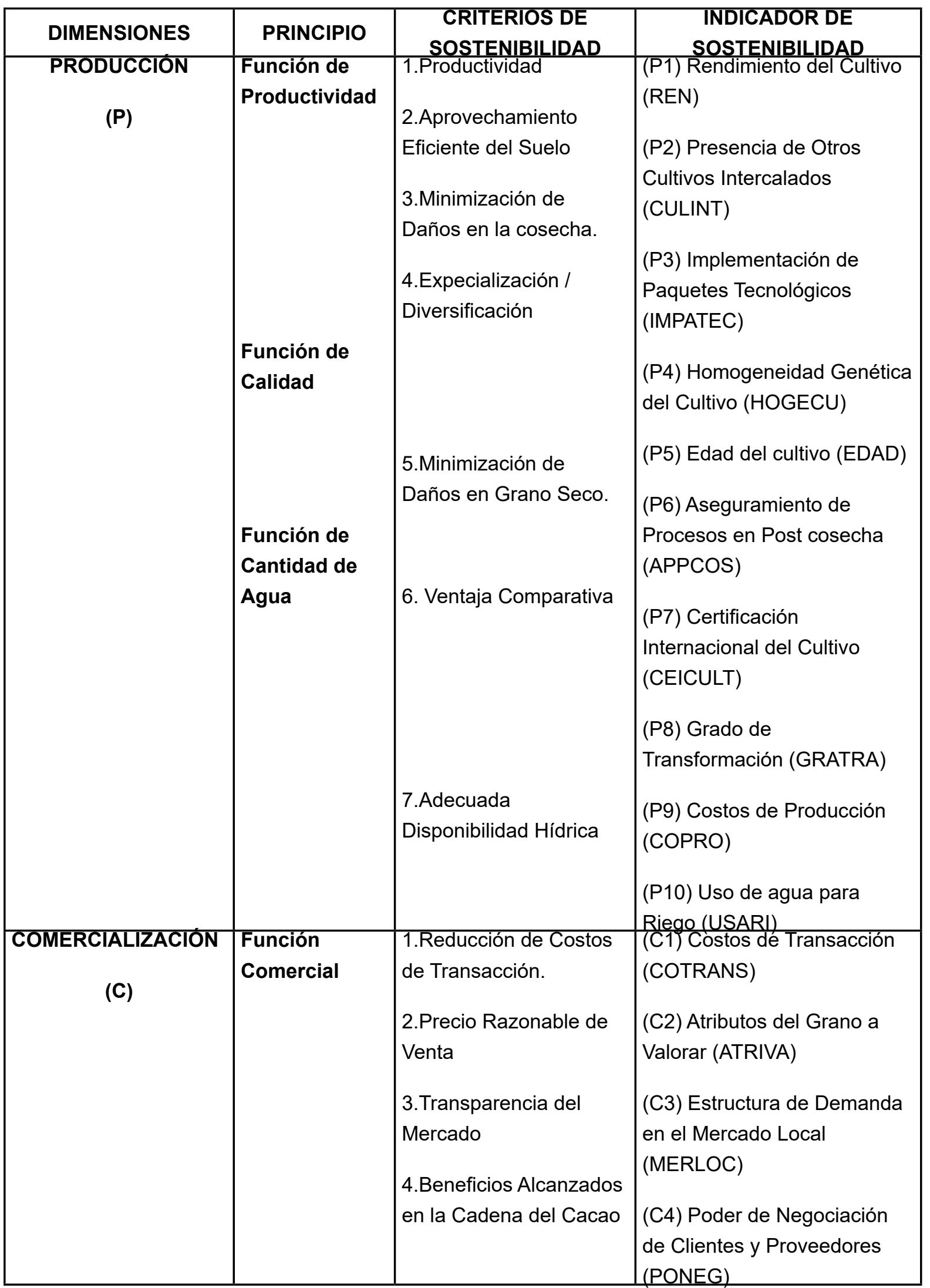




\begin{tabular}{|c|c|c|c|}
\hline $\begin{array}{c}\text { GESTION } \\
\text { (G) }\end{array}$ & $\begin{array}{l}\text { Función de } \\
\text { Gestión } \\
\\
\text { Función } \\
\text { Socioeconó- } \\
\text { mica }\end{array}$ & $\begin{array}{l}\text { 1.Nivel de Tecnificación } \\
\text { del Cultivo } \\
\text { 2. Acceso a Servicios } \\
\text { Públicos, Técnicos y } \\
\text { Financieros } \\
\text { 3.Capacitación en el } \\
\text { Manejo del Sistema } \\
\text { Productivo } \\
\text { 4.Adecuada } \\
\text { Administración del } \\
\text { Negocio } \\
\text { 5.Optimización de las } \\
\text { Condiciones de Trabajo } \\
\text { 6.Continuidad } \\
\text { Intergeneracional en la } \\
\text { Actividad } \\
\text { 7.Adecuada } \\
\text { Dependencia de la } \\
\text { Actividad Cacaotera } \\
\text { 8.Capacidad de Asumir } \\
\text { Compromiso Solidario y } \\
\text { Comunitario } \\
\text { 9.Garantía a una Renta } \\
\text { de los Productores. } \\
\text { 10.Minimización de } \\
\text { la Dependencia de } \\
\text { Subsidios } \\
\text { Proceso Productivo }\end{array}$ & $\begin{array}{l}\text { (G1) Inversión de Capital } \\
\text { en el Proceso Productivo } \\
\text { (INVCAP) } \\
\text { (G2) Asistencia Técnica, } \\
\text { Financiera y de Servicios } \\
\text { Públicos (ATFISEP) } \\
\text { (G3) Nivel Educativo y } \\
\text { de Formación Obtenida } \\
\text { (NIVEFO) } \\
\text { (G4) Plan de manejo } \\
\text { (PLAMA) } \\
\text { (G5) Capacidad de Generar } \\
\text { Empleo (CAGEM) } \\
\text { (G6) Riesgo de } \\
\text { Abandono de la Actividad } \\
\text { Cacaotera(RAACA) } \\
\text { (G7) Dependencia } \\
\text { Económica de la Actividad } \\
\text { Cacaotera (DE) } \\
\text { (G8) Participación en } \\
\text { asociaciones de productores } \\
\text { (ASOPRO) } \\
\text { (G9) Rentabilidad Financiera } \\
\text { del Cultivo (RENFI) } \\
\text { (G10) Subsidios recibidos } \\
\text { (SUBREC) }\end{array}$ \\
\hline
\end{tabular}

Fuente: Elaboración propia 
A partir del marco teórico revisado y panel realizado con expertos de Fedecacao y del Centro de Investigación Cenigaa, se logró recabar información técnica para identificar los 25 indicadores de sostenibilidad económica, que guardan consistencia y coherencia con los 6 principios y 22 criterios. Estos niveles jerárquicos, facilitarán la medición de los parámetros y el análisis empírico, para el logro del objetivo propuesto, que se describen en la Gráfica No 3, Gráfica No 4 y Gráfica No 5.

\section{Gráfica No 3 Interpretación para la Dimensión de Producción}

\begin{tabular}{|c|c|c|c|}
\hline No. & NOMBRE & DEFINICIÓN & INTERPRETACIÓN \\
\hline $\mathbf{P 1}$ & $\begin{array}{l}\text { Rendimiento del } \\
\text { cultivo }\end{array}$ & $\begin{array}{l}\text { Estimación de la cantidad de } \\
\text { grano seco producido, por } \\
\text { hectárea cosechada }\end{array}$ & $\begin{array}{l}\text { Menor a } 600 \text { k/h la sostenibilidad } \\
\text { agroeconómica está en riesgo. } \\
\text { Entre } 601 \text { k/h y } 900 \text { k/h sobrevive. } \\
\text { Entre } 901 \mathrm{k} / \mathrm{h} \text { y } 1.500 \mathrm{k} / \mathrm{h} \text { es buena. } \\
\text { Mayor a los } 1.500 \mathrm{k} / \mathrm{h} \text { suponen la } \\
\text { mavor sostenibilidad }\end{array}$ \\
\hline$\overline{\mathbf{P 2}}$ & $\begin{array}{l}\text { Presencia } \\
\text { de Cultivos } \\
\text { Intercalados }\end{array}$ & $\begin{array}{l}\text { Un sistema de producción } \\
\text { caracterizado por una sucesión } \\
\text { de cultivos de ciclo corto, } \\
\text { mediano y largo; de acuerdo } \\
\text { con cada etapa: instalación, } \\
\text { levante y producción }\end{array}$ & $\begin{array}{l}\text { La selección de especies de ciclo } \\
\text { corto en la etapa de instalación y } \\
\text { levante, mejoran la sostenibilidad } \\
\text { económica con sus cosechas. Las } \\
\text { agroforestales lo hacen con la } \\
\text { etapa de producción. }\end{array}$ \\
\hline P3 & $\begin{array}{l}\text { Tmplementación } \\
\text { de Paquetes } \\
\text { Tecnológicos }\end{array}$ & $\begin{array}{l}\text { Formulación de acciones } \\
\text { de carácter fitosanitario, } \\
\text { dosificaciones de nutrientes } \\
\text { y enmiendas para obtener un } \\
\text { balance apropiado de suelo y } \\
\text { planta }\end{array}$ & $\begin{array}{l}\text { La apropiación oportuna de } \\
\text { recomendaciones técnicas para } \\
\text { nutrientes, agua, desyerbas, podas, } \\
\text { control a plagas y enfermedades, } \\
\text { favorece una mayor sostenibilidad. }\end{array}$ \\
\hline$\overline{\mathbf{P 4}}$ & $\begin{array}{l}\text { Aseguramiento } \\
\text { de la Calidad en } \\
\text { Post cosecha. }\end{array}$ & $\begin{array}{l}\text { Procesos y procedimientos, } \\
\text { aprendidos y controlados para } \\
\text { la recolección, fermentación, } \\
\text { secado y almacenamiento del } \\
\text { grano. }\end{array}$ & $\begin{array}{l}\text { Capacidad de ofrecer con } \\
\text { una probabilidad } 100 \% \text {, } \\
\text { las características físicas, } \\
\text { organolépticas y de inocuidad, que } \\
\text { se requiere la norma. }\end{array}$ \\
\hline$\overline{\mathbf{P 5}}$ & $\begin{array}{l}\text { Homogeneidad } \\
\text { del cultivo }\end{array}$ & $\begin{array}{l}\text { Identificar y caracterizar las } \\
\text { especies de cacao explotadas. }\end{array}$ & $\begin{array}{l}\text { Entre mayor es la heterogeneidad } \\
\text { en el tipo de cacao, mayor es la } \\
\text { dificultad de clasificación del grano } \\
\text { y aumenta el costo de transacción. }\end{array}$ \\
\hline P6 & Edad del cultivo & $\begin{array}{l}\text { Los cultivos disminuyen su } \\
\text { productividad debido a efectos } \\
\text { lógicos de la edad, originando } \\
\text { renovación o rehabilitación }\end{array}$ & $\begin{array}{l}\text { Plantaciones que sobrepasen } \\
\text { la edad de } 25 \text { años, disminuyen } \\
\text { notablemente su productividad. } \\
\text { Renovar o rehabilitar posibilita un } \\
\text { mayor rendimiento. }\end{array}$ \\
\hline
\end{tabular}




\begin{tabular}{|l|l|l|l|}
\hline P7 & $\begin{array}{l}\text { Certificación del } \\
\text { cultivo }\end{array}$ & $\begin{array}{l}\text { Organizar el sistema productivo } \\
\text { para certificarlo con base en una } \\
\text { norma o criterio, para garantizar } \\
\text { productividad y rentabilidad. }\end{array}$ & $\begin{array}{l}\text { Gestionar una certificación } \\
\text { internacional abre nuevos } \\
\text { mercados y mejora la capacidad de } \\
\text { negociación. Lo orgánico, el origen } \\
\text { o BPA son, certificables. }\end{array}$ \\
\hline P8 & $\begin{array}{l}\text { Transformación } \\
\text { del grano }\end{array}$ & $\begin{array}{l}\text { Hacer transformación al grano, } \\
\text { mucílago o cáscara, para el } \\
\text { consumo final o como materia } \\
\text { prima para la industria. }\end{array}$ & $\begin{array}{l}\text { Gestionar procesos para generar } \\
\text { valor como: chocolates, cacao, } \\
\text { cacao tostado, manteca de cacao, } \\
\text { abono orgánico, incrementa la } \\
\text { sostenibilidad. }\end{array}$ \\
\hline P9 & $\begin{array}{l}\text { Costo de } \\
\text { producción }\end{array}$ & $\begin{array}{l}\text { Cuantificar el valor a pagar por } \\
\text { la ejecución de actividades del } \\
\text { sistema productivo como: mano } \\
\text { de obra, insumos, equipos y } \\
\text { herramientas, enmiendas y } \\
\text { productivos y administrativos, } \\
\text { buscando la racionalidad } \\
\text { económica, fomenta la } \\
\text { sostenibilidad para cada fase del } \\
\text { ciclo de vida del cultivo. }\end{array}$ \\
\hline P10 & Uso de agua de & $\begin{array}{l}\text { Determinar el balance hídrico } \\
\text { requerido para el cultivo de } \\
\text { cacao y la disponibilidad de } \\
\text { agua del sistema productivo. }\end{array}$ & $\begin{array}{l}\text { Entre mayor sea la cantidad de } \\
\text { agua de riego disponible en m3 / ha } \\
\text { mayor es la sostenibilidad. }\end{array}$ \\
& Riego &
\end{tabular}

Fuente: Elaboración propia 
Gráfica No. 4

\section{Dimensión de Comercialización}

\begin{tabular}{|c|c|c|c|}
\hline No. & NOMBRE & DEFINICIÓN & INTERPRETACIÓN \\
\hline C1 & $\begin{array}{l}\text { Costos de } \\
\text { transacción }\end{array}$ & $\begin{array}{l}\text { Determinar las condiciones } \\
\text { físicas, económicas y de } \\
\text { seguridad, para la recepción } \\
\text { de los insumos requeridos en } \\
\text { el proceso productivo y para la } \\
\text { entrega del cacao seco vendido. }\end{array}$ & $\begin{array}{l}\text { El estado de las vías terciarias y } \\
\text { principales, la situación política y } \\
\text { de seguridad, los acuerdos con } \\
\text { compradores y proveedores o } \\
\text { la legalización de contratos de } \\
\text { suministros, afectan los costos de } \\
\text { transacción. }\end{array}$ \\
\hline C2 & $\begin{array}{l}\text { Atributos del } \\
\text { grano a valorar }\end{array}$ & $\begin{array}{l}\text { Identificar y documentar las } \\
\text { características físicas, químicas } \\
\text { y organolépticas, así como las } \\
\text { prácticas de manipulación e } \\
\text { higiene del grano seco. }\end{array}$ & $\begin{array}{l}\text { El cumplimiento de normas de } \\
\text { calidad e inocuidad establecidas } \\
\text { por los clientes, favorece } \\
\text { directamente los resultados } \\
\text { económico de los productores de } \\
\text { cacao. }\end{array}$ \\
\hline C3 & $\begin{array}{l}\text { Estructura de } \\
\text { demanda en el } \\
\text { mercado local } \\
\text { específico }\end{array}$ & $\begin{array}{l}\text { Seleccionar el canal de } \\
\text { distribución más rentable, } \\
\text { influenciado por la industria } \\
\text { transformadora, los } \\
\text { comisionistas y el precio } \\
\text { internacional. }\end{array}$ & $\begin{array}{l}\text { Es la encargada de asegurar la } \\
\text { venta de las cosechas. Entre más } \\
\text { larga sea la cadena de distribución } \\
\text { menos ingresos netos percibe el } \\
\text { productor de cacao. }\end{array}$ \\
\hline $\mathrm{C4}$ & $\begin{array}{l}\text { Poder de } \\
\text { negociación } \\
\text { de clientes y } \\
\text { proveedores. }\end{array}$ & $\begin{array}{l}\text { Estimar el nivel de control que } \\
\text { ejercen sobre el productor los } \\
\text { clientes que compran el cacao y } \\
\text { los proveedores que suministran } \\
\text { los insumos. }\end{array}$ & $\begin{array}{l}\text { Cuanto mayor sea el poder } \\
\text { de negociación de clientes y } \\
\text { proveedores mayor será la } \\
\text { capacidad para negociar con el } \\
\text { productor exigiendo menores } \\
\text { precios de venta y altos precios en } \\
\text { los insumos. }\end{array}$ \\
\hline
\end{tabular}

Fuente: Elaboración propia 


\section{Gráfica No 5}

Interpretación para la Dimensión de Gestión

\begin{tabular}{|c|c|c|c|}
\hline No. & NOMBRE & DEFINICIÓN & INTERPRETACIÓN \\
\hline G1 & $\begin{array}{l}\text { Inversión de } \\
\text { capital en } \\
\text { el proceso } \\
\text { productivo }\end{array}$ & $\begin{array}{l}\text { Conocer la disposición } \\
\text { del productor para realizar } \\
\text { inversiones de capital, con } \\
\text { el objeto de mantener las } \\
\text { plantaciones de cacao existentes } \\
\text { o aumentarlas con nuevas, de } \\
\text { manera individual o a través de } \\
\text { fondos de capital colectivo. }\end{array}$ & $\begin{array}{l}\text { Invertir o reinvertir capital en } \\
\text { el mejoramiento del sistema } \\
\text { productivo, mejora los rendimientos, } \\
\text { la calidad y la competitividad., } \\
\text { con compensaciones por ser una } \\
\text { actividad generadora de tributos, } \\
\text { exenciones, exoneraciones y/o } \\
\text { deducciones. }\end{array}$ \\
\hline G2 & $\begin{array}{l}\text { Asistencia } \\
\text { técnica, financiera } \\
\text { y de servicios } \\
\text { públicos }\end{array}$ & $\begin{array}{l}\text { La apropiación de la tecnología } \\
\text { disponible para acceder al } \\
\text { crédito, la comercialización, el } \\
\text { control y la renovación del cultivo. }\end{array}$ & $\begin{array}{l}\text { Esta gestión protege la rentabilidad } \\
\text { y los riesgos de bajos rendimientos; } \\
\text { promueve el bienestar a las familias } \\
\text { cacaoteras. }\end{array}$ \\
\hline G3 & $\begin{array}{l}\text { Nivel educativo } \\
\text { y formación en } \\
\text { cada cultura. }\end{array}$ & $\begin{array}{l}\text { Establecer el nivel de estudios } \\
\text { de los titulares responsables } \\
\text { de sistema productivo, } \\
\text { relacionados con la agricultura } \\
\text { o en especialidades diferentes, } \\
\text { así como la formación en temas } \\
\text { específicos de la cacaocultura. }\end{array}$ & $\begin{array}{l}\text { Mientras los conocimientos } \\
\text { técnicos de los agricultores son } \\
\text { mayores, estos pueden gestionar } \\
\text { mejor los sistemas productivos del } \\
\text { cacao, haciéndolo más rentables y } \\
\text { sostenibles. }\end{array}$ \\
\hline G4 & Plan de manejo & $\begin{array}{l}\text { Una adecuada administración del } \\
\text { negocio para lograr eficiencias } \\
\text { con las técnicas de alto } \\
\text { rendimiento utilizada, requiere } \\
\text { contar con un plan de manejo. }\end{array}$ & $\begin{array}{l}\text { Gestionar el sistema productivo del } \\
\text { cacao con un plan documentado } \\
\text { conjuntamente con el asistente } \\
\text { técnico, permite hacer seguimiento y } \\
\text { control para el logro de los objetivos. }\end{array}$ \\
\hline G5 & $\begin{array}{l}\text { Capacidad de } \\
\text { generar empleo }\end{array}$ & $\begin{array}{l}\text { Se busca medir las implicaciones } \\
\text { sociales de la agricultura } \\
\text { del cacao en la provisión y } \\
\text { distribución de rentas a través del } \\
\text { empleo agrario. }\end{array}$ & $\begin{array}{l}\text { Mano de obra total cuantificada en } \\
\text { jornales de trabajo por hectárea. Un } \\
\text { mayor valor del indicador significa } \\
\text { que el cultivo tiene mayor capacidad } \\
\text { de generación de empleo. }\end{array}$ \\
\hline G6 & $\begin{array}{l}\text { Riesgo de } \\
\text { abandono } \\
\text { del actividad } \\
\text { cacaotera por } \\
\text { edad (RAE) y } \\
\text { renta (RAR) del } \\
\text { agricultor }\end{array}$ & $\begin{array}{l}\text { Establecer si el cultivo es el } \\
\text { resultado de una labor que se } \\
\text { ha realizado de generación en } \\
\text { generación y determinar el riesgo } \\
\text { de abandono de la actividad } \\
\text { productiva por edad y/o renta. }\end{array}$ & $\begin{array}{l}\text { El riesgo de abandono por edad } \\
\text { (RAE) se presenta, cuando el } \\
\text { agricultor con edad superior a los } \\
60 \text { años, no dispone de un sucesor } \\
\text { claro para su actividad. El riesgo de } \\
\text { abandono por renta (RAR) cuando la } \\
\text { renta es menor al } 50 \% \text { de la media } \\
\text { del sector en su comarca. }\end{array}$ \\
\hline
\end{tabular}




\begin{tabular}{|c|c|c|c|}
\hline G7 & $\begin{array}{l}\text { Dependencia } \\
\text { económica } \\
\text { de actividad } \\
\text { cacaotera }\end{array}$ & $\begin{array}{l}\text { Conocer si el cacao constituye } \\
\text { el cultivo principal de la unidad } \\
\text { productiva y si su cosecha es el } \\
\text { único medio de subsistencia }\end{array}$ & $\begin{array}{l}\text { Refleja el ingreso del titular del } \\
\text { cultivo derivados de la cosecha y el } \\
\text { grado de dedicación a la explotación } \\
\text { del cultivo. En la medida que el } \\
\text { indicador se acera más al } 100 \% \text {, se } \\
\text { reduce el riesgo de abandono. }\end{array}$ \\
\hline G8 & $\begin{array}{l}\text { Participación en } \\
\text { asociaciones } \\
\text { productoras }\end{array}$ & $\begin{array}{l}\text { Establecer el nivel de } \\
\text { asociatividad y el grado real de } \\
\text { participación en organizaciones } \\
\text { de productores. }\end{array}$ & $\begin{array}{l}\text { Hacer parte de una organización } \\
\text { asociativa y/o cooperativa de } \\
\text { productores, participando de las } \\
\text { decisiones, garantiza un mayor } \\
\text { gestión. }\end{array}$ \\
\hline G9 & $\begin{array}{l}\text { Rentabilidad } \\
\text { financiera del } \\
\text { cultivo }\end{array}$ & $\begin{array}{l}\text { Conocer qué tan atractiva } \\
\text { económicamente es la actividad } \\
\text { productiva del cacao, para la } \\
\text { inversión. Es la Gestión que } \\
\text { minimiza el uso de insumos } \\
\text { externos y maximiza el uso de } \\
\text { insumos internos. }\end{array}$ & $\begin{array}{l}\text { Se trata de establecer la viabilidad } \\
\text { financiera del cultivo. Si la } \\
\text { Rentabilidad financiera es positiva } \\
\text { y mayor a los costos del capital } \\
\text { el sistema es sostenible. Si es } \\
\text { negativa de manera sostenida no es } \\
\text { sostenible económicamente. }\end{array}$ \\
\hline G10 & $\begin{array}{l}\text { Subsidios } \\
\text { recibidos }\end{array}$ & $\begin{array}{l}\text { Resulta de interés conocer y } \\
\text { analizar, la incidencia en la } \\
\text { sostenibilidad agroeconómica, } \\
\text { a partir de variables de tipo } \\
\text { coyuntural de control de } \\
\text { los agricultores, como las } \\
\text { subvenciones acopladas a } \\
\text { la producción o a programas } \\
\text { agroambientales. }\end{array}$ & $\begin{array}{l}\text { Todas las subvenciones a la } \\
\text { producción, comercialización y } \\
\text { agroambientales, tienden a mejorar } \\
\text { la sostenibilidad socioeconómica } \\
\text { del sistema productivo. Se requiere } \\
\text { conocer si son significativas para la } \\
\text { sostenibilidad, en cada caso }\end{array}$ \\
\hline G11 & Innovación & $\begin{array}{l}\text { Conocer si el productor fomenta } \\
\text { la innovación en los procesos } \\
\text { productivos o emprendimientos } \\
\text { para la transformación del grano } \\
\text { o la cáscara. }\end{array}$ & $\begin{array}{l}\text { Entre mayor sea el porcentaje de } \\
\text { innovación o de emprendimiento, } \\
\text { hay mayor posibilidades para la } \\
\text { sostenibilidad agroeconómica. }\end{array}$ \\
\hline
\end{tabular}

Fuente: Elaboración propia

\section{RESULTADOS}

Existe un conjunto de marcos para evaluar la sostenibilidad agraria, que pretenden interpretarla bajo modelos de tipo normativos, sistémicos o analíticos, que sustenta un robusto cuerpo de conocimiento teórico y empírico, construido sobre la racionalidad de maximizar los beneficios en la interrelación de los recursos naturales y los socioeconómicos, como se aprecia en la Gráfica No 1, Los procesos para la medición van desde el ámbito nacional hasta el nivel local, siguiendo un modelo multiescalar, que puede seguir una ruta de análisis bottom-up (de "abajo a arriba") esto es un análisis deductivo o top-Down lo contrario, dada la interrelación con las decisiones cambiantes del mercado nacional e internacional del cacao y sus derivados. 
Gráfica No 1

PRINCIPALES MARCOS METODOLÓGICOS PARA EVALUAR LA SOSTENIBILIDAD

\begin{tabular}{|c|c|c|c|c|c|c|c|c|}
\hline MARCO & ENFOQUE & $\begin{array}{l}\text { DIMENSIÓN } \\
\text { EVALUADA }\end{array}$ & $\begin{array}{c}\text { PERIODO } \\
\text { DE } \\
\text { EVALUACIÓN }\end{array}$ & $\begin{array}{l}\text { ESCALA } \\
\text { DE } \\
\text { ANÁLISIS }\end{array}$ & $\begin{array}{c}\text { PROCESO } \\
\text { DE SELECCIÓN } \\
\text { DE INDICADORES }\end{array}$ & $\begin{array}{l}\text { INTEGRACIÓN } \\
\text { OBTENCIÓN DE } \\
\text { ÍNDICES }\end{array}$ & EVALUADORES & $\begin{array}{l}\text { GRADO } \\
\text { DE } \\
\text { UTILIZACIÓN }\end{array}$ \\
\hline $\begin{array}{l}\text { FESLM } \\
\text { (1993) }\end{array}$ & $\begin{array}{l}\text { Normativo } \\
\text { (orientado } \\
\text { A objetivos) }\end{array}$ & $\begin{array}{l}\text { Económica } \\
\text { Ambiental }\end{array}$ & ex-post & Nacional & 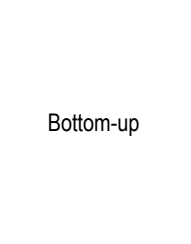 & $\begin{array}{l}\text { No integra } \\
\text { De fácil } \\
\text { adaptación }\end{array}$ & $\begin{array}{l}\text { Consultor ex } \\
\text { Actores } \\
\text { locales }\end{array}$ & $\begin{array}{l}\text { Alto } \\
\text { Dumanski (1994) } \\
\text { Gameda el al. (1997) } \\
\text { Vanloon et al. (2005) }\end{array}$ \\
\hline $\begin{array}{l}\text { IICA } \\
\text { (1993) }\end{array}$ & $\begin{array}{l}\text { Analítico } \\
\text { (relación } \\
\text { Causa / } \\
\text { Efecto) }\end{array}$ & $\begin{array}{l}\text { Económica } \\
\text { Ambiental }\end{array}$ & ex-post & Institucional & Top-down & No Integra & $\begin{array}{l}\text { Consultor } \\
\text { Externo }\end{array}$ & Bajo \\
\hline $\begin{array}{l}\text { PSR y } \\
\text { DPSIR } \\
\text { (1994 y } \\
1995)\end{array}$ & $\begin{array}{l}\text { Analítico } \\
\text { (relación } \\
\text { Causa / } \\
\text { Efecto) }\end{array}$ & Ambiental & ex-post & $\begin{array}{l}\text { Nacional } \\
\text { Regional }\end{array}$ & Bottom-up & $\begin{array}{l}\text { Índice } \\
\text { Agregado }\end{array}$ & $\begin{array}{l}\text { Consultor } \\
\text { Externo }\end{array}$ & $\begin{array}{l}\text { Alto } \\
\text { OCDE, } 2001 \\
\text { IRENA, } 2006\end{array}$ \\
\hline $\begin{array}{l}\text { CIFOR } \\
(1999)\end{array}$ & $\begin{array}{l}\text { Normativo } \\
\text { (orientado } \\
\text { A objetivos) }\end{array}$ & $\begin{array}{l}\text { Económica } \\
\text { Ambiental }\end{array}$ & ex-post & $\begin{array}{l}\text { Nacional } \\
\text { Regional }\end{array}$ & $\begin{array}{l}\text { Top-down } \\
\text { Bottom-up }\end{array}$ & No Integra & $\begin{array}{l}\text { Consultor ex } \\
\text { Actor local }\end{array}$ & $\begin{array}{l}\text { Alto } \\
\text { Estudios } \\
\text { Manejo de bosques }\end{array}$ \\
\hline $\begin{array}{r}\text { MESMIS } \\
\text { (1999) }\end{array}$ & $\begin{array}{l}\text { Normativo } \\
\text { (orientado } \\
\text { A objetivos) }\end{array}$ & $\begin{array}{l}\text { Económica } \\
\text { Ambiental } \\
\text { Social }\end{array}$ & ex-post & $\begin{array}{l}\text { Sis. Agrario } \\
\text { Explot. } \\
\text { Agraria }\end{array}$ & Bottom-up & $\begin{array}{l}\text { Integra } \\
\text { Gráficos } \\
\text { Amoeba }\end{array}$ & $\begin{array}{l}\text { Consultor } \\
\text { Externo }\end{array}$ & $\begin{array}{l}\text { Muy Alto } \\
\text { Comunidades } \\
\text { Campesinas } \\
\text { Masera (1999) }\end{array}$ \\
\hline $\begin{array}{l}\text { INSURE } \\
(2005)\end{array}$ & Sistémico & $\begin{array}{l}\text { Económico } \\
\text { Ambiental } \\
\text { Social } \\
\text { Institucional }\end{array}$ & ex-post & Regional & Top-down & $\begin{array}{l}\text { Integra (uso } \\
\text { De gráfico pizza) }\end{array}$ & $\begin{array}{l}\text { Consultor } \\
\text { Externo }\end{array}$ & $\begin{array}{l}\text { Bajo } \\
\text { Consultorias }\end{array}$ \\
\hline $\begin{array}{l}\text { SAFE } \\
(2007)\end{array}$ & $\begin{array}{l}\text { Normativo } \\
\text { (orientado a } \\
\text { objetivos) }\end{array}$ & $\begin{array}{l}\text { Económica } \\
\text { Ambiental } \\
\text { Social }\end{array}$ & ex-post & $\begin{array}{l}\text { Sis. Agrario } \\
\text { Explot. } \\
\text { Agraria } \\
\text { Parcela }\end{array}$ & $\begin{array}{l}\text { Top-down } \\
\text { Bottom-up }\end{array}$ & Integra & $\begin{array}{l}\text { Consultor ext. } \\
\text { Actores locales }\end{array}$ & $\begin{array}{l}\text { Sauvenier (2006) } \\
\text { Sánchez (2009) }\end{array}$ \\
\hline $\begin{array}{l}\text { GESE } \\
(2021)\end{array}$ & $\begin{array}{l}\text { Normativo } \\
\text { (orientado } \\
\text { A objetivos) }\end{array}$ & $\begin{array}{l}\text { Económica } \\
\text { Social }\end{array}$ & ex-post & $\begin{array}{l}\text { Sis. Agrario } \\
\text { Explot. } \\
\text { Agraria } \\
\text { Parcela }\end{array}$ & $\begin{array}{l}\text { Top-down } \\
\text { Bottom-up }\end{array}$ & Integra & $\begin{array}{l}\text { Investigador } \\
\text { Alberto } \\
\text { Ducuara } \\
\text { Manrique }\end{array}$ & $\begin{array}{l}\text { Desconocido (marco } \\
\text { en construcción). }\end{array}$ \\
\hline
\end{tabular}


En la Gráfica No 1 se registran los factores relevantes que ofrecen las principales metodologías utilizadas para evaluar la sostenibilidad agraria, que no agotan las posibilidades de encontrar respuestas a las necesidades en diferentes casos espacio temporales de cultivos específicos, como el caso del marco propuesto para el cacao GESE, a nivel de la parcela.

\section{MARCO GESE}

El marco para la Gestión de la Sostenibilidad Económica del cacao (GESE), se basa en una representación propia, que propicia una interpretación sistémica de las unidades productivas de cacao. Es un concepto cultural que se orienta al logro de objetivos humanos, económicos y sociales, destinado a adaptarse a las necesidades de los cacaoteros, en cualquier momento y localidad en particular. Ver Gráfica No 7.

\section{Gráfica No 7 MARCO GESE}

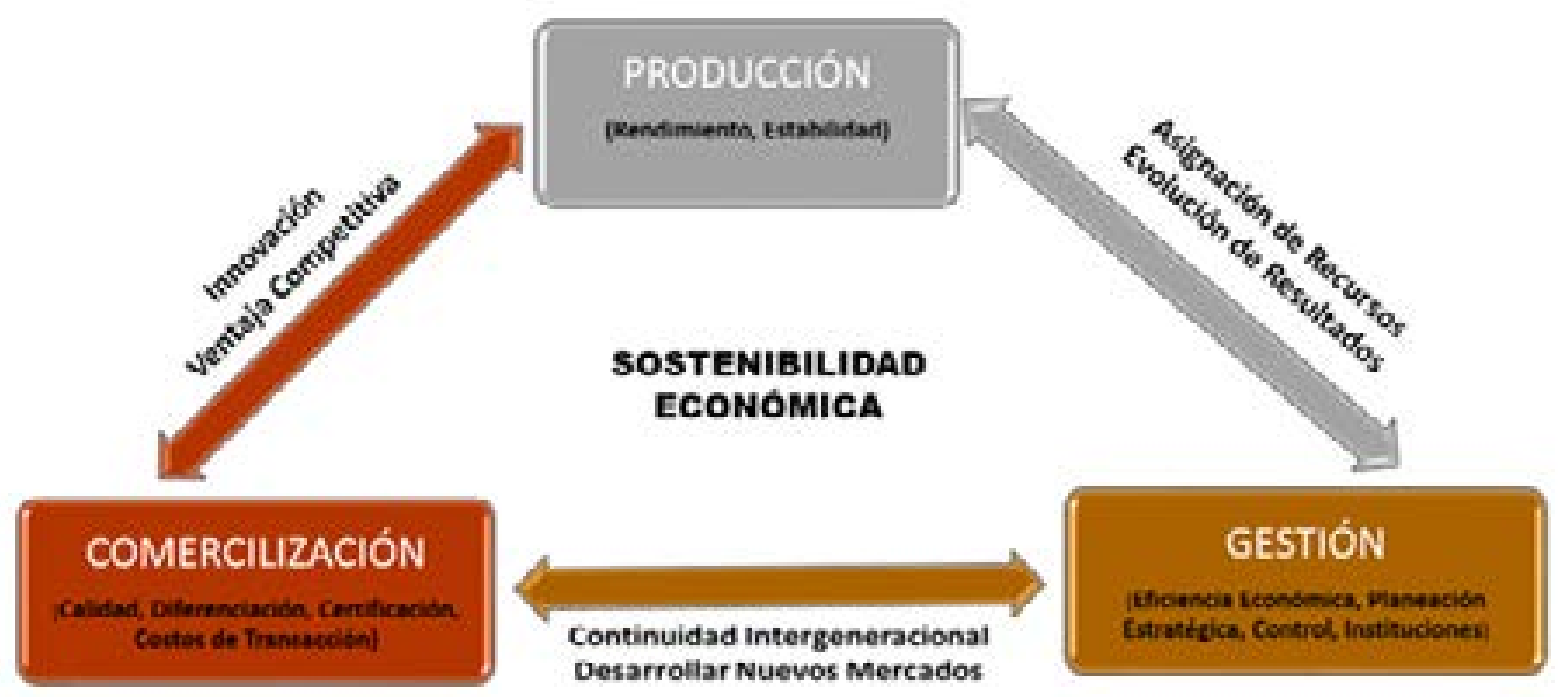

Fuente: Elaboración propia.
La dimensión de la Producción es la responsable de generar los granos de cacao, a partir de la interrelación del capital natural, capital humano, capital financiero y capital tecnológico. Se visualiza a partir de los rendimientos, la calidad, la innovación, los residuos, el aporte ambiental, el empleo. Estos resultados son valorados a través de indicadores, por el productor, el mercado y la sociedad.

La dimensión de comercialización representa la valoración que hace el mercado, de la producción en términos de cantidad, calidad, costos de transacción, trazabilidad, confianza y capacidad de negociación del productor, todo influenciado por la regulación gubernamental, la demanda y la oferta.

La dimensión de gestión está relacionada con la racionalidad organizativa e instrumental, derivadas de las prácticas administrativas de la planeación, asignación de recursos, el control y la innovación. Se encarga de alcanzar los mínimos económicos viables, que permita sostener la producción, las ventas y demás servicios asociados al sistema productivo del cacao, para generar una rentabilidad financiera apropiada. 
Para formular la propuesta de sostenibilidad económica, el proceso de planificación se hace con base en tres pilares para el sistema de producción, previamente definidos: Gestión, Comercialización y Producción, ver Gráfica No 2. Se prevé un enfoque de tipo normativo soportado en objetivos, en periodos de evaluación de la sostenibilidad ex - post, con una escala de análisis a nivel de la parcela de producción, que se somete a una aplicación empírica. Respecto a su definición, esta se sintetiza en el siguiente enunciado propio:
"La Sostenibilidad Económica del cacao, es un constructo social de aprendizaje, capaz de gestionar y evaluar el sistema productivo, aprovechando las cambiantes oportunidades del entorno económico y la oferta ambiental, para obtener una viabilidad económica de manera sostenida en el tiempo".

La parcela de producción, que constituye la unidad para las decisiones, está circunscrita en un sistema productivo, donde se pueden identifican las diferentes variables de insumo, proceso y resultado, que determinan el universo de donde se seleccionarán los indicadores base. Ver Gráfica No 8.

\section{Gráfica No 8 SISTEMA DE PRODUCCIÓN DE CACAO}

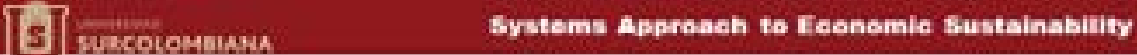

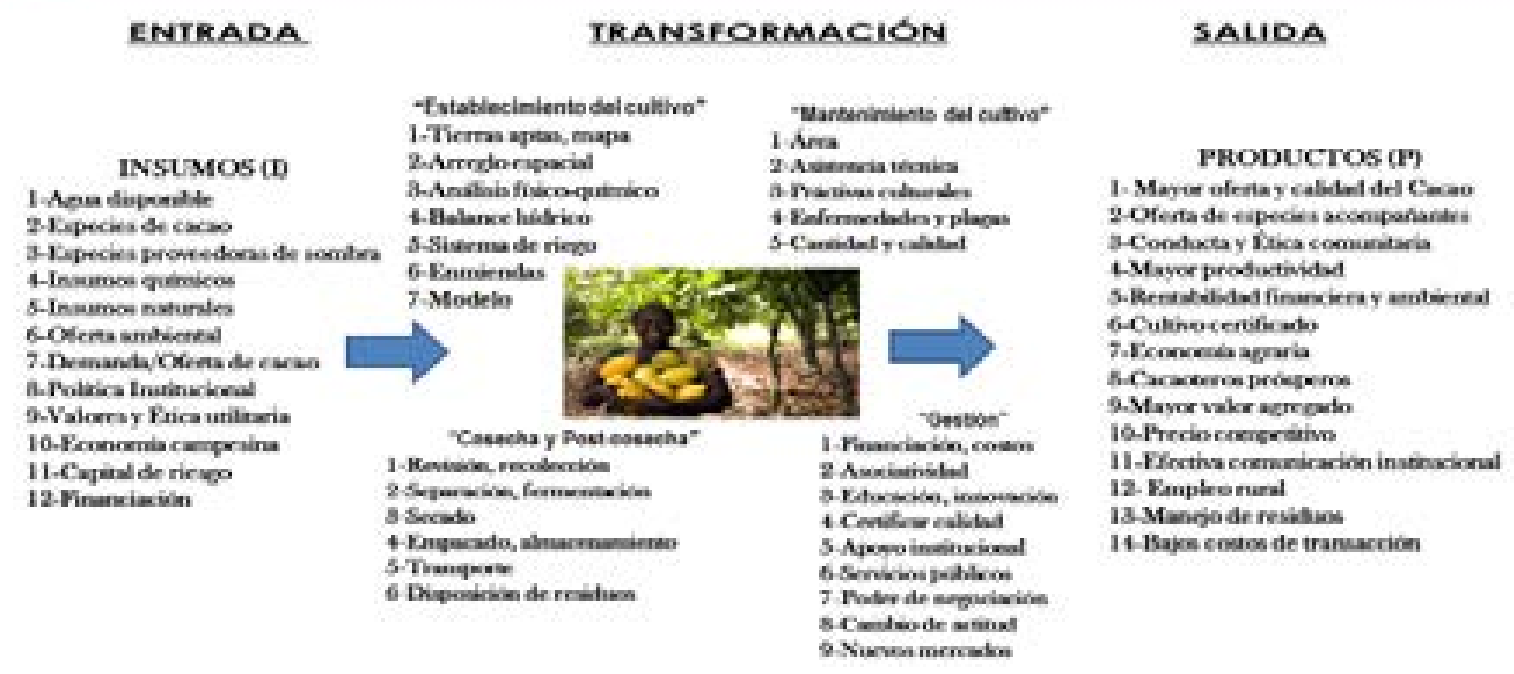

Fuente: Elaboración propia

La función de valuación de salidas del sistema, concibe escenarios para la sostenibilidad, cuando el valor neto de las salidas obtenidas no disminuye con el tiempo $\mathrm{V}(\mathrm{Pt}+1) \geq \mathrm{V}(\mathrm{Pt})$, por lo tanto, la gestión del sistema productivo de cacao, con fines productivos más allá de la producción de subsistencia, se relaciona con estrategias para la generación de cambios en el sistema y poder mejorar las salidas $\mathrm{Pt}+1=\mathrm{f}(\mathrm{St}$, It).

Dicha gestión, está medada por las capacidades dinámicas para la sostenibilidad económica, liderada por los productores. Se aborda desde la perspectiva de la contingencia, que se define como las capacidades que permiten responder a los cambios producidos en el entorno (Eisenhard \& Martin, 2000). Se trata de capacidades para 
identificar estrategias de gestión proactivas, que responden a un esquema del tipo señales externas, desarrollando un proceso que incluye interpretación, respuesta y evaluación.

Se pretende encontrar formas organizativas del cacao, a través de un plan como el propuesto en la Gráfica No 9, haciendo énfasis en las orientaciones significativas, las habilidades para adaptar el sistema productivo y ejercer un desempeño, para alcanzar resultados iguales a superiores al mínimo vital para la sostenibilidad, con una connotación económica de mantener el stock de capital total.

\section{Gráfica No 9. PLAN PARA LA GESTIÓN DE LA SOSTENIBILIDAD ECONÓMICA DEL CACAO}

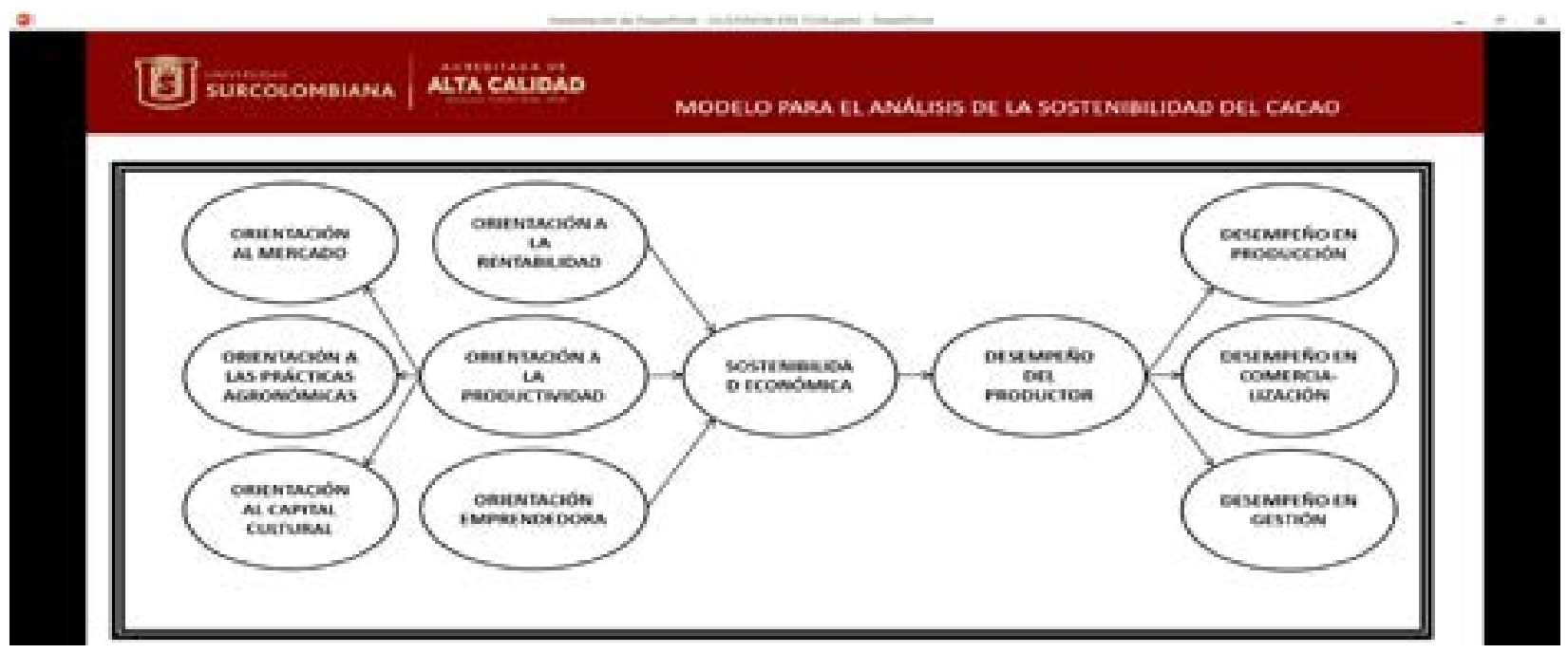

Fuente: Elaboración propia.

En este proceso de previsión de respuestas a los cambios del mercado y la oferta ambiental, se propone el análisis de la sostenibilidad de las explotaciones agrarias de cacao mediante indicadores, como elemento de apoyo para mejorar la gobernanza del sector.

\section{CONCLUSIONES:}

- La metodología de medición de la sostenibilidad a través de indicadores e índices, puede ofrecer una utilidad práctica y posibilidad real de aplicación, en la medida en que integra variables representativas del sistema productivo en las dimensiones producción, comercialización y gestión.

- Identificar las variables en cada
dimensión, guían la construcción de los indicadores e índices, que pueden evaluar y monitorear el sistema productivo del cacao durante la vida útil y comparar varios sistemas de manejo diferenciados.

- El marco metodológico propuesto, busca estimar con una alta probabilidad los mínimos viables en renta y bienestar, de los productores de cacao en un espacio y una temporalidad.

- El enfocarnos en la problemática de la sostenibilidad económica, promueve el que otros problemas comenzarán a resolverse al mismo tiempo: pobreza rural, distribución inequitativa de la riqueza y protección del tejido social, entre otros, por lo que es potencialmente útil para la gobernanza del sector. 


\section{REFERENCIAS BIBLIOGRÁFICAS}

Barrezueta, S., \& Paz, A. (2018). Indicadores de sostenibilidad social y económico: caso productores de cacao en el Oro, Ecuador. Ciencia, 20-29.

Boisier, S. (2005). ¿Hay espacio para el desarrollo local en la globalización? CEPAL, 47-62.

Cárdenas, G. I., Giraldo, H., Idárraga, Á., \& Vásquez, L. N. (2006). Desarrollo y Validación de Metodología para Evaluar con indicadires la Sustentabilidad de Sistemas Productivos Campesinos de la Asociación de Caficultores Orgánicos de Colombia. UNISARC: ACOC.

Cuba, O. H. (2017). Cacao. Producción, consumo y comercio. Del período prehispánico a la actualidad en América Latina. Fronteras de la Historia Vol. 22 $N^{\circ} 1$.

Darnhofer, I., Gibbon, D., \& Dedieu, B. (2012). Methodological themes in farming systems research and implications for learning in higher education. En I. Darnhofer, D. Gibbon, \& B. Dedieu, Farming Systems Research Into The 21st Century: The New Dynamic (págs. 95 - 115). Londres: Springer.

Eisenhard, K., \& Martin, J. (2000). DYNAMIC CAPABILITIES: WHAT ARE THEY? Strategic Management Journal, 11051121.

Flora, C., Flora, J. L., \& Gasteyer, S. P. (2018). Rural Communities. Legacy and Change. New York: Taylor \& Francis Group.
Gotsch, N., \& Burger, K. (02 de 05 de 2001). Dynamic Supply Response and Welfare Effects of Technological Change on Perennial Crops: The Case of Cocoa in Malaysia. Recuperado el 20 de 10 de 2018, de https://academic.oup.com/ ajae/article-abstract/83/2/272/55990

Hansen, J. (1995). Is Agricultural Sustainability a Useful Concept? Elsevier, 117-143.

Hernandez, R., Fernández, C., \& Baptista, P. (2010). Metodología de la Investigación . México: Mc Graw Hill.

Lammerts van Bueren, E., \& Blom, E. (1996). Hierarchical Framework for the Formulation of Sustainable Forest Management Standars. Tropenbos International, 1-45.

Martínez, A. (1986). La Sombra en el cacao: revision de la literatura y bibliografía anotada. Costa Rica: Centro Agronómico Tropical de Investigación y Enseñanza, CATIE.

Masera, O., Astier, M., \& López, S. (2000). Sustentabilidad y Manejo de Recursos Naturales. México: Mundi-Prensa México, S.A. de C.V.

OECD, \& JRC. (2008). Handbook on Constructing Composite Indicators. Methodology ans user guide. Ispra - Italia: OECD.

Ramirez, J. A., Sigarroa, A. K., \& Del Valle, R. (2014). Characterization of Cocoa (Theobroma cacao L.) Farming Systems un the Norte de Santander Department and Assessment of Their Sustainability. Facultad Nacional de Agronomía. Universidad Nacional, 7177 - 7187. 
Ramirez, R., Guevara, F., D’Armas, M., Pena, R., Farias, R., \& Bravo, F. (2017). Análisis Multivariante: Teoría y práctica de las principales técnicas. Milagro - Ecuador: Ediciones Holguin S.A.

Sanchez, G. (2009). Tesis Doctoral. Análisis de la sostenibilidad agraria mediante indicadores sintéticos: aplicación empírica para sistemas agrariosde Castilla y León. Madrid - España: Universidad Politécnica de Madrid.

Strange, T., \& Bayley, A. (2012). Desarrollo sostenible. Integrar la economía, la sociedad y el medio ambiente. México: Insttituto de Investigaciones Económicas de la UNAM.

Torres, P., Rodriguez, L., \& Sanchez, O. (2004). Eavluación de la sustentabilidad del desarrollo regional. El marco de la agricultura. SciElo Analytics, 1-15.

Vilches, A., Gil, D., \& Toscano, J. (10 de Octubre de 2014). Economía y Sostenibilidad. Obtenido de http://www.researchgate. net/publication/302293071 